\title{
Multi-element network reveals the mystery of species adaptation and coexistence
}

\author{
Jiahui Zhang ${ }^{1}$, Tingting Ren ${ }^{2}$, Junjie Yang ${ }^{3}$, Li Xu${ }^{1}$, Mingxu Li $^{4}$, Yunhai Zhang ${ }^{5}$, \\ Xing-Guo $\mathrm{Han}^{6}$, and Nianpeng $\mathrm{He}^{7}$ \\ ${ }^{1}$ Institute of Geographic Sciences and Natural Resources Research, Chinese Academy of \\ Sciences \\ ${ }^{2}$ Institute of Botany, Chinese Academy of Sciences \\ ${ }^{3}$ Institute of Botany Chinese Academy of Sciences \\ ${ }^{4}$ Institute of Geographic Sciences and Natural Resources Research Chinese Academy of \\ Sciences \\ ${ }^{5}$ State Key Laboratory of Vegetation and Environmental Change, Institute of Botany, The \\ Chinese Academy of Sciences, Beiijing CN \\ ${ }^{6}$ State Key Laboratory of Vegetation and Environmental Change \\ ${ }^{7}$ Institute of Geographical Sciences and Natural Resources Research, Chinese Academy of \\ Sciences
}

May 5, 2020

\begin{abstract}
Plant biochemical reactions are dependent on the combined action of multiple elements. However, it remains unclear how these elements co-vary to adapt to environmental change. Here, we propose a novel concept of the multi-element network (MEN) including the mutual effects between elements to more effectively explore the alterations in response to long-term nitrogen (N) deposition simulations. MENs were constructed with 18 elements and were species specific. Macroelements were more stable, but microelements were more susceptible to $\mathrm{N}$ deposition. Interestingly, higher MEN plasticity determined increased relative aboveground biomass (species importance) for different species in one functional group under simulated $\mathrm{N}$ deposition. Furthermore, the association between MEN plasticity and species importance was consistently verified along a dry-wet transect. In summary, MENs provide a novel approach for exploring the adaptation strategies of plants and to better predict community composition under altering nutrient availability or environmental stress associated with future global climate change.
\end{abstract}

\section{Hosted file}

20200216-multiple element network-mainfile_zjh.docx available at https://authorea.com/users/ 299099/articles/428496-multi-element-network-reveals-the-mystery-of-species-adaptationand-coexistence 